\title{
PANDEMIA Y TELETRABAJO EN BRASIL
}

\section{Giselle Gonzalez Gonçalves ${ }^{1}$}

Sumario: Introducción. I.- El lugar de trabajo como definidor de la naturaleza de la prestación. Il.- Trabajo a distancia y legislación brasileña. III.- Pandemia en Brasil y trabajo fuera del entorno del empleador. IV.- Legislación de emergencia - MP 927/20 e MP 1.046/2021. V.-Conclusión

Resumen: El presente estudio tiene como objetivo exponer la realidad legislativa frente al fenómeno del trabajo a distancia en Brasil, abordándose críticamente la actual regulación del instituto del teletrabajo. Además, se plantean los diferentes conceptos de los distintos tipos de trabajo a distancia y sus consecuencias en el marco jurídico y en la relación laboral.

Palabras clave: Teletrabajo, trabajo a distancia, flexibilización de la jornada, falta de regulación, precarización.

\section{I.- Introducción}

El distanciamiento social, impuesto como medida de contención a la propagación del coronavirus, ha traído a la sociedad el reto de adaptarse a una rutina extraordinaria a muchos sectores de la economía mundial. Las actividades laborales que nunca fueron concebidas para ser realizadas a distancia se vieron obligadas a ceder a esta modalidad, no tan nueva de prestación de servicios.

Los empresarios brasileños nunca fueron muy adeptos al trabajo a distancia, ya que, culturalmente, la presencia física del trabajador en el ambiente de trabajo y la posibilidad de controlar la jornada laboral por parte del empleador "validan" la ejecución efectiva del trabajo.

En este sentido, hasta el advenimiento de la Reforma Laboral de 2017, no existía un marco legal que abarcara esta práctica con detalles.

La ley $n^{\circ}$ 13.467/2017, Ley de la Reforma Laboral, a pesar de numerosos aspectos controvertidos, incluso en relación con la constitucionalidad de algunos institutos, trajo las primeras líneas que rodean la regulación de la modalidad de teletrabajo en Brasil, que ha sido de máximo valor para el contexto actual de pandemia, aunque el instituto no se encuentre suficientemente desarrollado como se verá a continuación. 
Cabe señalar que en Brasil el porcentaje de trabajadores a domicilio saltó de 3,8 millones de personas en 2018 a 7,9 millones en abril de 2021 (IBGE, 2021); y hay una tendencia de pensar que algunas actividades tradicionales no retomaran de forma completa a la presencialidad dados los resultados positivos obtenidos a lo largo de este inesperado laboratorio forzado (REVISTA ISTO É, 2020).

\section{I.- El lugar de trabajo como definidor de la naturaleza de la prestación}

El acto de trabajar fuera del entorno del empleador se puede nombrar de varias maneras: "trabajo remoto", "trabajo a distancia", "teletrabajo", "oficina en casa/home office" entre otros. Aunque estas expresiones puedan parecer sinónimas, existen sutiles diferencias que dan un significado especial a la forma en que se prestan los servicios y pueden no reconocer la aplicación de una norma particular a un caso concreto si se comprueba que, en la práctica, el trabajo realizado bajo una determinada modalidad no cumple con los requisitos establecidos en la ley.

El "trabajo remoto" o "trabajo a distancia" acentúa la lejanía del servicio prestado, que puede realizarse desde cualquier lugar ajeno a las instalaciones del empleador. El "teletrabajo" enfatiza el uso de las TIC - Tecnologías de la Información y la Comunicación. EI "home office" destaca la tarea que se desarrolla en la residencia habitual del trabajador.

Oficialmente la OIT utiliza el término "trabajo a domicilio" en su Convenio 177 y en la Recomendación 184 correspondiente sobre el tema. Lo definió como la producción de bienes o servicios realizados por un individuo en su hogar o lugar de su elección, a cambio de un salario, bajo la especificación de un empleador o intermediario (OIT, 1996). Es importante destacar que la principal preocupación de este documento internacional al formular esa definición era hacer hincapié en el factor "subordinación" en la relación entre el contratista y el contratado y no necesariamente hacer hincapié en el lugar donde se desarrollaría la actividad.

En este sentido, es posible notar el malentendido en el uso de la expresión "trabajo a domicilio", ya que el propio tratado internacional se refiere claramente al trabajo realizado fuera del entorno del empleador y no sólo al trabajo realizado en el hogar del empleado.

En consecuencia, dentro de una clasificación terminológica sería posible definir el "trabajo a distancia" o "trabajo remoto" como un género, cuyas especies serían "teletrabajo" y "oficina en casa/home office".

Específicamente en Brasil, como se abordará más adelante, la diferenciación de expresiones es una medida que se impone, ya que el modus operandi de cada tipo de prestación de servicios a distancia, fuera del entorno del empleador puede influir en la incidencia o no de la legislación ordinaria existente, ya que hasta ahora, sólo se regula el teletrabajo.

\section{II.-Trabajo a distancia y legislación brasileña}

Fue en 2011, con la llegada de la Ley 12.551, que el trabajo realizado a distancia se equiparó al trabajo presencial mediante la modificación del artículo 6 de la CLT (Consolidação das Leis do Trabalho).

Así, no se distingue entre el trabajo realizado en el establecimiento del empleador, el realizado en el domicilio del empleado y el realizado a distancia, siempre que se carac- 
tericen los supuestos legales de la relación laboral. En cuanto a los medios telemáticos e informatizados de mando, control y supervisión, éstos se equiparan a efectos de subordinación jurídica, a los medios personales y directos de mando, control y supervisión del trabajo. En ese momento, la ecuación fue vista como un avance, aunque aún no era una regulación específica sobre el tema (ROCHA y AMADOR, 2018).

Pero más tarde, en 2017, la Reforma Laboral fue responsable de introducir en la legislación brasileña el concepto de teletrabajo, añadiendo al artículo 75 de la CLT las letras A, B, C, D y E, en el capítulo II - A, "TELETRABAJO".

Aunque esta ley es objeto de duras críticas derivadas de su finalidad y aprobación, se encargó de definir el teletrabajo, establecer su forma de ejecución y gestión de los activos y mencionar una posible limitación a la responsabilidad del empleador por los riesgos laborales.

Se considera "teletrabajo" en Brasil la prestación de servicios predominantemente fuera de las instalaciones del empleador, con el uso de tecnologías de la información y la comunicación que, por su naturaleza, no constituyen trabajo externo y la asistencia a los locales del empleador para la realización de actividades específicas que requieren la presencia del empleado no descaracteriza el régimen de teletrabajo (Artículo 75 - B de la CLT).

En cuanto a la forma, la adopción del teletrabajo sólo puede llevarse a cabo mediante un contrato de trabajo individual, es decir, un contrato escrito, que debe especificar las actividades que llevará a cabo el empleado (artículo 75 - C de la CLT). El cambio contractual del régimen presencial para el teletrabajo se puede realizar de mutuo acuerdo entre las partes, registrado como un anexo contractual (artículo 75-C, párrafo 1 de la CLT), lo contrario también puede suceder por el cumplimiento del período mínimo de transición de 15 días también con un anexo contractual.

Por lo que se refiere a los bienes utilizados para la prestación de servicios de teletrabajo, establece el siguiente texto que la responsabilidad de la adquisición, mantenimiento o suministro de los equipos e infraestructuras tecnológicas necesarios y adecuados, así como el reembolso de los gastos a cargo del empleado, deben estar previstos en un contrato escrito (artículo $75 \mathrm{D}$ de la CLT) y estos servicios mencionados no forman parte de la remuneración del empleado (artículo 75-D, párrafo único de la CLT).

La ley también establece que el empleado debe firmar un término de responsabilidad comprometiéndose a seguir las instrucciones proporcionadas por el empleador con respecto a los riesgos del desarrollo de actividades laborales (artículo 75 - E, párrafo único de la CLT).

Y, en cuanto al control jornada, la inclusión del teletrabajo en la CLT también provocó una alteración en el artículo 62, una vez que fue introducido el ítem III que estableció expresamente que no hay control de jornada para los trabajadores en teletrabajo. En otras palabras, a los dependientes del régimen de teletrabajo no se les deben horas extraordinarias.

En resumen, para que la prestación de servicios a distancia sea considerada modalidad de teletrabajo en Brasil es necesario la utilización de tecnologías de la información y de comunicación; además de la existencia de un contrato escrito que demuestre la opción a esta modalidad que también prevea la responsabilidad de la adquisición de bienes y servicios, y establezca la posibilidad de reembolso por los gastos domiciliarios extras. 
Como se puede observar, la norma que regula la modalidad del teletrabajo es sumamente formal, por ende, la falta de uno de los requisitos por ella establecido desnaturaliza el instituto. ${ }^{2}$

\section{III.- Pandemia en Brasil y trabajo fuera del entorno del empleador}

La implementación masiva y forzada del trabajo a distancia en Brasil es un gran desafío aún más por tratarse de un país que abarca las más distintas realidades sociales y financieras entre sus ciudadanos.

Ocurre que después del registro del primer caso de Covid-19 diagnosticado oficialmente el 26/02/2020 en la ciudad de São Paulo (MINISTÉRIO DA SAÚDE, 2021), los sectores productivos vieron la necesidad primaria de poner en práctica el distanciamiento social en el ambiente de trabajo. Sin embargo, como ya se ha sugerido, el trabajo a distancia, en su forma amplia, no tiene regulación en Brasil ya que solamente el teletrabajo está regulado hasta este momento.

En este sentido, el cambio del lugar de prestación de servicio del empleado -de los locales de la empresa a su residencia- por sí mismo, por sí mismo, no caracteriza la adopción del teletrabajo en la forma establecida en el texto legal (CLT), si, obviamente, no se cumplen los demás requisitos previstos en la referida reglamentación.

En este sentido, está aún más evidente el vacío legislativo con respecto a la regulación de otras formas de trabajo fuera del entorno de la empresa, actividades que no se caracterizan por la definición de teletrabajo.

Por otra parte, hubo cierta preocupación por parte de algunos parlamentarios con el mantenimiento de los textos originales de los artículos 75 -D y 62, III de la CLT, que tratan respectivamente de la responsabilidad por la adquisición de bienes y materiales para la ejecución del teletrabajo y la ausencia de control de jornada, ya que una reflexión más atenta nos permitió vislumbrar un posible carácter nocivo para el trabajador que pudieran contener estos artículos.

Ante este panorama, se presentaron a la Cámara de Diputados proyectos de ley que pretenden modificar el texto actual de la CLT; siendo el más relevante el PL 3915/2020 que prevé la adopción de normas para el reembolso de materiales y otros gastos con teletrabajo, PL 5282/2020 que propone el control de la jornada laboral para teletrabajadores y PL 2251/2020 que propone la regulación del trabajo en casa/home office.

\section{IV.-Legislación de emergencia - MP 927/20 eMP 1.046/2021}

Con la intención de "regular" y flexibilizar el texto de la CLT sobre teletrabajo para su aplicación durante la pandemia del Coronavirus, el gobierno dictó medidas extraordinarias: MP 927/20 (válido del 22/03/2020 al 09/07/2020) y MP 1.046/2021 (plazo 28/04/2021 al 25/08/2021).

Ambas medidas prevén sobre la implementación de la prestación de servicios en las

\footnotetext{
2 Es importante mencionar que el Poder Ejecutivo a través de la Medida Provisional 927/2020, flexibilizó las normas contenidas en la CLT para la implementación de la modalidad de teletrabajo durante la pandemia de Coronavirus. Este acto presidencial hizo posible el teletrabajo unilateral por parte del empleador, sin registro en el contrato (teniendo como único requisito la comunicación a lo empleado 48 horas antes del inicio de la prestación de servicios). También preveía la posibilidad de celebrar un acuerdo entre el trabajador y el empresario para la adquisición y el suministro de maquinaria, además del reembolso de gastos. MP 927/2020 entró en vigor del 22 de marzo de 2020 y fue hasta el 31 de julio de 2020 y no fue convertida en ley a través del proceso legislativo. El 2021, la MP 927/2020 fue reeditada bajo la MP 1.046/2021.
} 
modalidades: "teletrabajo", trabajo remoto u otro tipo de trabajo a distancia, ampliando y equiparando a los tres conceptos distintos bajo el mismo entendimiento - lo que no es regulado por la norma (CLT) originalmente, ya que su texto sólo se refiere al teletrabajo.

Críticas aparte, la MP 1.046/2021, que no es más que la reedición de la MP 927/20, suprimió la necesidad de documentación del cambio de la modalidad presencial para el teletrabajo, permitiendo que el cambio por acto discrecional del empleador se comunicara al empleado con una antelación de 48 horas, y también le dio al empleador la obligación de proporcionar al empleado - que no disponga del equipo tecnológico necesario o de la infraestructura necesaria y adecuada para ejecución del trabajo a distancia - los medios necesarios para realizarlo. La legislación de emergencia ha receptado el régimen de teletrabajo, trabajo remoto o trabajo a distancia para aprendices, lo no estaba permitido hasta entonces.

\section{V.-Conclusión}

Si consideramos que la afirmación "el futuro del trabajo es el trabajo remoto" es cierta, también es cierta la necesidad de regulación del instituto, dado que, hasta hoy, el trabajo a distancia o remoto, como el género, no ha sido regulado en Brasil.

Por otra parte, una cuestión que merece ser destacada es la falta de singularidad conceptual en la referencia a la prestación de servicios fuera del entorno del empleador, ya que existe, al menos en Brasil, un desorden en el uso de las expresiones "trabajo a distancia", "trabajo remoto", "teletrabajo", "trabajo en casa", "oficina en casa", como es posible verificar incluso a través del texto la MP 1.046/21. En este sentido, la definición de cada una de las modalidades también es urgentemente necesaria para proporcionar una mayor seguridad jurídica en sus implementaciones.

En cuanto a la regulación existente del teletrabajo, un punto de gran controversia es la falta de control de jornada.

Ahora bien, si el teletrabajo requiere como condición previa el uso de las tecnologías de la información y la comunicación, las llamadas TICS, ¿cómo no se puede realizar a través de ellas el control de jornada?

Es evidente que las TICs proporcionan el control de jornada laboral aunque el trabajador se encuentre fuera de las instalaciones de la empresa, y en este sentido, es inadmisible la prolongación de la jornada laboral sin la debida indemnización. Esta situación está siendo verificada diariamente durante la actual crisis sanitaria en algunos sectores (CORREIO BRAZILIENSE, 2020). No parece creíble que el legislador, en el momento de redactar la ley, no previó voluntariamente una situación para ello.

Por último, es notorio que queda mucho por hacer en el ámbito de la ley para que situaciones concretas y reiteradas sean positivisadas, más aún en el momento actual, que requiere cautela y prudencia tanto en las relaciones laborales como en la economía, para que el legado dejado por la pandemia del Coronavirus no sea tan difícil de mitigar. 


\section{Bibliografía.}

- BRASIL. CONSOLIDAÇÃO DAS LEIS DO TRABALHO (CLT). Disponível em <http://www. planalto.gov.br/ccivil_03/decreto-lei/del5452.htm>. Último acceso: 1 de marzo de 2021.

- BRASIL. MP 927/2020. Disponível em < http://www.planalto.gov.br/ccivil_03/_ato20192022/2020/mpv/mpv927impressao.htm>. Último acceso: 1 de marzo de 2021.

- BRASIL. MP 1.046/2021. Disponível em < https://www.in.gov.br/en/web/dou/-/medida-provisoria-n-1.046-de-27-de-abril-de-2021-316265470>. Último acceso: 4 de julio de 2021.

- CÂMARA DOS DEPUTADOS. PL 2251/2020. Disponível em < https://www.camara.leg.br/ proposicoesWeb/fichadetramitacao?idProposicao=2250778،. Último acceso: 1 de marzo de 2021.

- CÂMARA DOS DEPUTADOS. PL 3915/2020. Disponível em «https://www.camara.leg.br/ proposicoesWeb/fichadetramitacao?idProposicao=2258556) Último acceso: 14 de abril de de 2021.

- CÂMARA DOS DEPUTAdOS. PROJETO PREVÊ CONTROLE DE JORNADA POR SISTEMA INFORMATIZADO NO TELETRABALHO. Disponível em <https://www.camara.leg.br/ noticias/712155-projeto-preve-controle-de-jornada-por-sistema-informatizado-no-teletrabalho/>. Último acceso: 18 de abril de 2021.

- CORREIO BRAZILIENSE. MAIS TRABALHO, MAIS SACRIFÍCIOS: OS EFEITOS DA PANDEMIA NO TRABALHADOR. Disponível em <https://www.correiobraziliense.com.br/euestudante/trabalho-e-formacao/2020/08/4872125-mais-trabalho--mais-sacrificios--os-efeitosda-pandemia-no-trabalhador.html>. Último acceso: 1 de marzo de 2021.

- IBGE - INSTITUTO BRASILEIRO DE GEOGRAFIA E ESTATÍSTICA. O IBGE APOIANDO O COMBATE À COVID-19. Disponível em <https://covid19.ibge.gov.br/pnad-covid/>. Último acceso: 1 de marzo de 2021.

- MINISTÉRIO DA SAÚDE. RESPOSTA NACIONAL E INTERNACIONAL DE ENFRENTAMENTO AO NOVO CORONAVÍRUS. Disponível em «https://coronavirus.saude.gov.br/linha-dotempo/s. Último acceso: 12 de abril de 2021.

- MINISTÉRIO DA SAÚDE. BLOG DA SAÚDE: 10 ANOS DO SURTO GLOBAL DE H1N1. Disponível em < http://www.blog.saude.gov.br/index.php/53845-10-anos-do-surto-global-deh1n1>. Último acceso: 13 de mayo de 2021.

- OIT - ORGANIZAÇÃO INTERNACIONAL DO TRABALHO. CONVENIO SOBRE EL TRABAJO A DOMICILIO, 1996 (NÚM. 177). Disponível em <https://www.ilo.org/dyn/normlex/es/f?p=N ORMLEXPUB:12100:0::NO::P12100_INSTRUMENT_ID:312322>. Último acceso: 1 de marzo de 2021.

- OMS - ORGANIZAÇÃO MUNDIAL DA SAÚDE. ACTUALIZACIÓN OFICIAL COVID-19. Disponível em <https://www.who.int/emergencies/diseases/novel-coronavirus-2019>. Último acceso: 1 de marzo de 2021.

- PORTAL G1. CRESCE O INTERESSE POR FORMAÇÃO À DISTÂNCIA DURANTE A PANDEMIA. Disponível em https://g1.globo.com/mg/centro-oeste/especial-publicitario/unicesumar/ educacao-a-distancia/noticia/2021/02/23/cresce-o-interesse-por-formacao-a-distanciadurante-a-pandemia.ghtml. Último acceso: 18 de abril de 2021.

- REVISTA ISTO É DINHEIRO. O FUTURO DO TRABALHO É REMOTO. Disponível em <https:// www.istoedinheiro.com.br/o-futuro-do-trabalho-e-remoto/>. Último acceso: 1 de marzo de 2021.

- REVISTA PEGN. Revista Pequenas Empresas Grandes Negócios. CRISE FECHOU 50 MIL EMPRESAS DO SETOR DE TURISMO EM TODO O PAÍS, DIZ ESTUDO. Disponível em < https://revistapegn.globo.com/Banco-de-ideias/Diversao-e-turismo/noticia/2020/10/ crise-fechou-50-mil-empresas-do-setor-de-turismo-em-todo-o-pais-diz-estudo.html.. Úl- 
timo acceso: 1 de marzo de 2021.

- REVISTA VEJA SAÚDE. GRIPE: QUAIS FORAM AS MAIORES EPIDEMIAS DA HISTÓRIA, 2020. Disponível em <https://saude.abril.com.br/medicina/gripe-quais-foram-as-maiores-epidemias-da-historia/>. Último acceso: 1 de marzo de 2021.

- ROCHA, Cháris Telles Martins da; AMADOR, Fernanda Spanier. O TELETRABALHO: CONCEITUAÇÃO E QUESTÕES PARA ANÁLISE. Cad. EBAPE.BR, Rio de Janeiro, v. 16, n. 1, p. 152-162, Jan. 2018. Disponível em <http://www.scielo.br/scielo.php?script=sci_ arttext\&pid=S1679-39512018000100152\&lng=en\&nrm=iso>. Último acceso: 18 de abril de 2021. https://doi.org/10.1590/1679-395154516.

- SENADO FEDERAL. TELETRABALHO GANHA IMPULSO NA PANDEMIA MAS É OBJETO DE CONTROVÉRSIA. Disponível em <https://www12.senado.leg.br/noticias/infomaterias/2020/07/teletrabalho-ganha-impulso-na-pandemia-mas-regulacao-e-objeto-de-controversia>. Último acceso: 1 de marzo de 2021.

- WORLDMETER. CORONAVIRUS AND POPULATION. Disponível em https://www.worldometers.info/br/. Último acceso: 1 de marzo de 2021. 